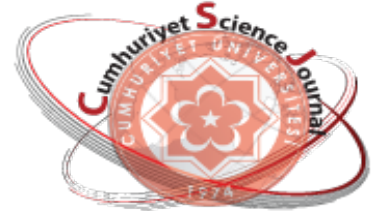

e-ISSN: $2587-246 X$

ISSN: $2587-2680$

\section{Cumherriyet Science Dournal es?}

Cumhuriyet Sci. J., Vol.39-1(2018) 34-46

\title{
A New Outlook for Almost Convergent Sequence Spaces
}

\author{
Murat CANDAN \\ Inönü University, Faculty of Art and Sciences, Department of Mathematics, Malatya-44280, TURKEY \\ Received: 24.01.2018; Accepted: 27.02.2018 \\ http://dx.doi.org/10.17776/csj.383311
}

\begin{abstract}
The point standing out in the present paper is the sequence spaces $\hat{c}_{0 G}^{\widetilde{B}}, \hat{c}_{G}^{\widetilde{B}}$ and $\hat{c} s_{G}^{\widetilde{B}}$ produced by the domain of the infinite matrix $\widetilde{R}=G \cdot \widetilde{B}$, which is defined in the previous study of Candan [2], where the spaces $\hat{c}_{0}, \hat{c}$ and $\hat{c} s$, respectively, are as presented by G.G. Lorentz utilizing the issue of the Banach limits (Acta. Math. 80. 1948, 167-190), and $\widetilde{B}$ is the double sequential band matrix and $\mathrm{G}$ is the generalized weighted mean. Firstly, it is shown that aforementioned spaces are linearly isomorhic to the spaces $\hat{c}_{0}, \hat{c}$ and $\hat{\mathcal{C}} S$, respectively. In addition to these, $\gamma$-and $\beta$-duals of the spaces $\hat{\mathcal{C}}_{G}^{\widetilde{B}}$ and $\hat{\mathcal{C}} s_{G}^{\widetilde{B}}$ are given. Beyond them, the classes $\left(\hat{c}_{G}^{\widetilde{B}}: \lambda\right)$ and $\left(\lambda: \hat{c}_{G}^{\widetilde{B}}\right)$ of infinite matrices are characterized, where $\lambda$ is a given sequence space. Keywords: Almost convergence; Generalized weigheted mean; Sequence space; Matrix transformations.
\end{abstract}

\section{Hemen Hemen Yakınsak Dizi Uzaylar için Yeni Bir Bakış}

Özet: Banach limiti (Acta. Math. 80. 1948, 167-190) kavramını kullanarak G.G. Lorentz hemen hemen yakınsak dizilerin $\hat{c}$ uzayını tanımladı. Bu çalışmada öne çıkan nokta $\hat{c}_{0}, \hat{c}$ ve $\hat{c} S$ uzaylarının Candan [2] tarafindan tanımlanan $\widetilde{R}=G . \widetilde{B}$ matris etki alanında olan $\hat{c}_{0 G}^{\widetilde{B}}$, $\hat{c}_{G}^{\widetilde{B}}$ ve $\hat{c} s_{G}^{\widetilde{B}}$ uzaylarını tanımlamaktır. Burada $\widetilde{B}$ ikili dizisel band matrisi $G$ de genelleştirilmiş ağırlıklı ortalamayı göstermektedir. Çalışmada öncelikle $\hat{c}_{0 G}^{\widetilde{B}}, \hat{c_{G}}$ ve $\hat{c} s_{G}^{\widetilde{B}}$ uzaylarının sirası ile $\hat{c}_{0}, \hat{c}$ ve $\hat{c} s$ uzaylarına lineer izomorf oldukları gösterildikten sonra $\hat{c}_{G}^{\widetilde{B}}$ ve $\hat{c} S_{G}^{\widetilde{B}}$ uzaylarının sırası ile $\gamma$-ve $\beta$-dualleri elde edilmiştir. Son bölümde de $\lambda$ verilen herhangi bir dizi uzayı olmak üzere $\left(\hat{c}_{G}^{\widetilde{B}}: \lambda\right)$ ve $\left(\lambda: \hat{c}_{G}^{\widetilde{B}}\right)$ matris sinıflarının karekterizasyonu verilmiştir.

Anahtar Kelimeler: Hemen hemen yakınsaklık; Genelleştirilmiş ağırlıklı ortalama; Dizi uzayı; Matris Dönüşümleri.

\footnotetext{
* Corresponding author. Email address: murat.candan@inonu.edu.tr http://dergipark.gov.tr/csj C2016 Faculty of Science, Cumhuriyet University
} 


\section{INTRODUCTION}

At the beginning of the introduction; first of all, the basic ideas about summability theory and historical background are given. Then the sequence spaces $\hat{c}_{0 G}^{\widetilde{B}}, \quad \hat{c}_{G}^{\widetilde{B}}$ and $\hat{c} s_{G}^{\widetilde{B}}$ which constitute the main body of this study, are presented. Begin with the definition of the sequence space, which is the fundamental concept of this study. As known, the symbol $w$ denotes the space of all real-valued sequences. Also, we use the symbols $c, c_{0}, l_{\infty}$ and $l_{p}$ $(1 \leq p<\infty), b s$ and $c s$ to present the sets of all, convergent, null, bounded sequences, $p$-absolutely convergent series, bounded series and convergent series, respectively. The sets defined in the above paragraph are vector spaces and at the same time those sets are subspace of $w$. Since any subspace of $w$ is known as a sequence space, each of aforementioned sets is a sequence space. In addition to these, it is going to be used the conventions that $e=(1,1, \cdots)$ and $e^{(n)}$ are the sequences whose only non-zero term is 1 and found in the $n^{\text {th }}$ position for each $n \in I N$, where $I N=\{0,1,2, \cdots\}$. We note that here $a=\left(a_{k}\right)$ and $X=\left(x_{n k}\right)$ instead of $a=\left(a_{k}\right)_{k=0}^{\infty}$ and $X=\left(x_{n k}\right)_{n, k=0}^{\infty}$, respectively.

Let $\lambda$ and $\mu$ be any subspace of $w$ and $A=\left(a_{n k}\right), n, k \in I N$, be infinite matrix of real or complex numbers. For any sequence $x=\left(x_{k}\right), \quad$ we write $A x=\left((A x)_{n}\right), \quad$ if $(A x)_{n}=\sum_{k} a_{n k} x_{k}$ converges for all $n \in I N$. If $x \in \lambda$ implies that $A x \in \mu$, we say that $A$ defines a matrix trasformation from $\lambda$ into $\mu$ and we denote it by $A: \lambda \rightarrow \mu$. By $(\lambda, \mu)$ or $(\lambda ; \mu)$ we denote class of matrices such that $A: \lambda \rightarrow \mu$.

Another essential definition we need is that the triangle matrix. Let us give this definition of that. A matrix $D=\left(d_{n k}\right)$ is called triangle if primary diagonal's components aren't equal to zero and components on the top of the primary diagonal are equal to zero. If we remember that given two matrices are triangle matrices then following equality is valid

$$
A(B . x)=(A \cdot B) x
$$

for a given sequence $x$. Additionally, a triangle matrix $A$ uniquely has an inverse $A^{-1}=B$ which is also a triangle matrix. Therefore, $x=A(B x)=B(A x)$ holds for all $x \in w$.

Now, it is time to give definition of Schauder basis in a normed space. Let $(X,\|\|$.$) be a normed$ space. A sequence $\left(e_{k}\right)$ in $X$ is called a Schauder basis for $X$ if for every element $x \in X$ there exist a unique sequence $\left(\vartheta_{k}\right)$ composed of scalars such that $x=\sum_{k=1}^{\infty} \vartheta_{k} e_{k}$, i.e.,

$$
\lim _{n \rightarrow \infty}\left\|x-\sum_{k=0}^{n} \vartheta_{k} e_{k}\right\|_{X}=0 .
$$

In that case, the series $\sum_{k=1}^{\infty} \vartheta_{k} e_{k}$ having the same $x$ is then called the expansion of $x$ with respect to $\left(e_{n}\right)$, and we write $x=\sum_{k} \vartheta_{k} e_{k}$.

Another point in this study is the matrix domain which is one of the most important concept. Because of this importance, let us now explain this concept. When an infinite matrix $A$ and a 
sequence space $\chi$ are given, the following newly defined set using both $A$ and $\chi$;

$$
\chi_{A}=\left\{x=\left(x_{k}\right) \in w: A x \in \chi\right\}
$$

is called the matrix domain of $\chi$ under $A$. This newly defined set is a vector space and is a subset of $w$ at the same time. Therefore, $\chi_{A}$ is known as a sequence space. It is worth mentioning here that in some cases or rather some special cases, even if $\chi_{A}$ and $\chi$ are equal, but usually one covers the other. Those interested about in the subtleties of this subject can consult especially on the $51^{\text {th }}$ page of Başar's book [17] and references at the end of the study for further information.

Building a new one using previously defined spaces is a common way of lately. Researchers interested in this subject should examine the relevant articles [5-13], [16], [18], [24-53] to gain different perspectives in the references.

The reader will do well to read about $K$-space, $F K$-space, $B K$-space, $A K$-space and $A D$-space of a sequence space and related topics before starting a systematic study about the present subject.

It is useful to state here that the terms map, function and transformation are all synonymous.

\section{THE MATRIX $\widetilde{R}$ and ALMOST CONVERGENCE}

Here, we focus on the new sequence spaces $\hat{c}_{0 G}^{\widetilde{B}}$, $\hat{\mathcal{C}}_{G}^{\widetilde{B}}$ and $\hat{\mathcal{C}} s_{G}^{\widetilde{B}}$ obtained by the domain of the infinite matrix $\widetilde{R}=G \cdot \widetilde{B}$ of the spaces $\hat{c}_{0}, \hat{c}$ and $\hat{c} S$, respectively, where $\widetilde{R}$ is defined in the previous study of Candan [2]. A full detail of the above aforementioned matrix $\widetilde{R}$ are important for us to work out at this time and we will just accept as valid without details the usual rules. For this approach, the article of Candan [2] is recommended.

Before giving some results, let us present a very brief historical background and developments about the space of almost convergent sequences.

We know that there are different impressions in the same sense about the space of almost convergent sequences. For example, one of these notations is $f$ and the other is $\hat{c}$, of the space of almost convergent sequences. We have chosen to use the representation of $\hat{c}$ in this study because $f$ is also used for other concepts. As everyone knows very well, the space $\hat{c}$ was presented by G. G. Lorentz [3], using the concept of the Banach limits defined immediately below.

Now, let us define an operator known as the shift operator from $w$ to $w$ as follows

$$
\wp_{n}(x)=x_{n+1}
$$

for all $n \in I N$. In the literature, $L$, which is defined on $l_{\infty}$, is known Banach limit, which is also a non-negative linear functional satisfying the following conditions $L(\wp \circ x)=L(x)$ and $\mathrm{L}(e)=1$. We all the sequence $x=\left(x_{k}\right) \in l_{\infty}$ almost convergent to the generalized limit $\varepsilon$ when all Banach limits of $x$ is equals $\varepsilon$ [3], and denote it by $\hat{c}-\lim x_{k}=\varepsilon$. Let $\wp^{j}$ be the composition of $\wp$ with itself $j$ times and define $k_{m n}(x)$ for a sequence $x=\left(x_{k}\right)$ by

$$
k_{m n}(x)=\frac{1}{m+1} \sum_{j=0}^{m} \wp_{n}^{j}(x) \text { for all } m, n \in I N .
$$

It has been proved by Lorentz in [3] that $\hat{c}-\lim x_{k}=\varepsilon$ if and only if 
$\lim _{m \rightarrow \infty} k_{m n}(x)=\varepsilon$ uniformly in $n$. It is known that every convergent sequence is almost convergent and both limits are equal. By $\hat{c}$ and $\hat{c} s$, we denote the space of all almost convergent sequences and series, in other words;

$\hat{c}=\left\{x \in w: \lim _{m \rightarrow \infty} k_{m n}(x)=l\right.$, uniformly in $\left.n\right\}$

$\hat{c} s=\left\{x \in w: \lim _{m \rightarrow \infty} \sum_{k=0}^{m} \sum_{j=0}^{n+k} \frac{y_{j}}{m+1}=l\right.$, uniformly in $\left.n\right\}$.

The inclusion $c \subset \hat{c} \subset l_{\infty}$ are strictly hold [3].

Because of these relations among them, norms \|\|$_{\hat{c}}$ and \|\|$_{\infty}$ of the spaces $\hat{c}$ and $l_{\infty}$ are equal. Therefore, these spaces $\hat{c}$ and $\hat{c}_{0}$ are $B K-$ spaces with the following norm $\|x\|_{\hat{c}}=\sup _{m, n}\left|k_{m n}(x)\right|$.

We are going to denote by $U$ the set of all sequences $u$ for which $u_{k} \neq 0$ for every $k \in I N$.

For $u \in U$, let $1 / u=\left(1 / u_{k}\right)$. Let us suppose that $u, v \in U$ and describe the matrices $G(u, v)=\left\{g_{n k}\right\} \quad$ which is one of the main matrices in this paper and known generalized weighted mean or factorable matrix is defined as follows:

$$
g_{n k}=\left\{\begin{array}{cc}
u_{n} v_{k}, & k<n \\
u_{n} v_{n}, & k=n \\
0, & k>n
\end{array}\right.
$$

for all $k, n \in I N$, where $u, v \in U$, also $u_{n}$ depends only on $n$ and $v_{k}$ bounds up with only $k$.

Now, let us start giving the definition of double band matrix $\widetilde{B}=B\left(r_{n}, s_{n}\right)$. Here and thereafter, for any given two sequences $\widetilde{r}=\left(r_{n}\right)$ and $\widetilde{s}=\left(s_{n}\right)$ the terms of which are positive real numbers; we are going to assume that these sequences are convergent. Let us describe the sequential band matrix $B(\widetilde{r}, \widetilde{s})=\left\{b_{n k}(\widetilde{r}, \widetilde{s})\right\}$ by

$$
b_{n k}(\widetilde{r}, \widetilde{s})=\left\{\begin{array}{ccc}
r_{n} & , & k=n \\
s_{n} & , & k=n-1 \\
0 & , & 0 \leq k<n-1 \text { or } k>n
\end{array}\right.
$$

for each $k, n \in I N$. It can be understood in a simple way, the generalized difference matrix $B(r, s)$ is obtained by making a special selection in the band matrix $B(\widetilde{r}, \widetilde{s})$, with a clearer expression; when $r_{n}=r$ and $s_{n}=s$ are taken for all $n \in I N$.

Now, suppose that $u=\left(u_{k}\right), v=\left(v_{k}\right) \in U$, $\left(r_{n}\right)$ and $\left(s_{n}\right)$ are defined immediately in the above page, two convergent sequences of positive real numbers. Now, we are going to use the notation $\widetilde{R}$ to represent the matrix $R(u, v, \widetilde{B})$ defined below as we did in previous.

$$
\widetilde{R}=R(u, v, \widetilde{B})=G(u, v) \cdot B(\widetilde{r}, \widetilde{s})
$$

and

$$
\Omega_{k}^{j}=\frac{1}{r_{j}}\left[\frac{1}{v_{k}} \prod_{i=k}^{j-1}\left(-\frac{s_{i}}{r_{i}}\right)-\frac{1}{v_{k+1}} \prod_{i=k+1}^{j-1}\left(-\frac{s_{i}}{r_{i}}\right)\right]
$$

where; $j, k \in I N$.

Now, it is time to give the definition sequence space $\hat{c}_{G}^{\widetilde{B}}$ by

$$
\hat{c}_{G}^{\widetilde{B}}=\left\{x=\left(x_{k}\right) \in w: y=\left(y_{k}\right)=(\widetilde{R} \cdot x)_{k} \in \hat{c}\right\}
$$


where the frequently used sequence $y=\left(y_{k}\right)$ by the $\widetilde{R}=R(u, v, \widetilde{B})$-transform of a given sequence $x=\left(x_{k}\right)$, that is $y_{0}=u_{0} v_{0} r_{0} x_{0}$ and for all $k \geq 1$

$y_{k}=u_{k}\left[\sum_{j=0}^{k-1}\left(r_{j} v_{j}+s_{j} v_{j+1}\right) x_{j}+v_{k} x_{k} r_{k}\right]$.

Similarly; we are also able to define the space $\hat{c} s_{G}^{\widetilde{B}}$ as follows;

$\hat{c} s_{G}^{\widetilde{B}}=\left\{x=\left(x_{k}\right) \in w: y=\left(y_{k}\right)=(\widetilde{R} \cdot x)_{k} \in \hat{c} s\right\}$.

We can redefine the spaces $\hat{c}_{0 G}^{\widetilde{B}}, \hat{c}_{G}^{\widetilde{B}}$ and $\hat{c} s_{G}^{\widetilde{B}}$ by the notion of (1.1)

$$
\hat{c}_{0 G}^{\widetilde{B}}=\left(\hat{c}_{0}\right)_{\widetilde{R}}, \hat{c}_{G}^{\widetilde{B}}=(\hat{c})_{\widetilde{R}} \text { and } \hat{c} s_{G}^{\widetilde{B}}=(\hat{c} S)_{\widetilde{R}} \text {. }
$$

Theorem 2.1. The sets $\hat{c}_{0 G}^{\widetilde{B}}, \hat{c}_{G}^{\widetilde{B}}$ and $\hat{c} s_{G}^{\widetilde{B}}$ are linear space.

Proof. Since this result is something familiar to the basic linear algebra, details are omitted.

Theorem 2.2. i) The sequence spaces $\hat{c}_{G}^{\widetilde{B}}$ is normed space with

$\|x\|_{\hat{c}_{G}^{\tilde{B}}}=\sup _{m, n}\left|\frac{1}{m+1} \sum_{k=0}^{m} u_{n+k}\left[\sum_{i=0}^{n+k+1} k_{i} x_{i}+v_{n+k} r_{n+k} x_{n+k}\right]\right|$

ii) The sequence space $\hat{c} S_{G}^{\widetilde{B}}$ is normed space with

$\|x\|_{\hat{c} S_{G}^{\tilde{B}}}=\sup _{m, n}\left|\frac{1}{m+1} \sum_{k=0}^{m}\left\{\sum_{j=0}^{k+n}\left(u_{j}\left[\sum_{i=0}^{j-1} k_{i} x_{i}+v_{j} r_{j} x_{j}\right]\right)\right\}\right|$

where $k_{i}=v_{i} r_{i}+v_{i+1} s_{i+1}$.
Theorem 2.3. The spaces $\hat{c}_{0 G}^{\widetilde{B}}, \hat{c}_{G}^{\widetilde{B}}$ and $\hat{c} s_{G}^{\widetilde{B}}$ are linearly isomorphic to the sets $\hat{c}_{0}, \hat{c}$ and $\hat{c} s$, respectively. With another representation in the same sense $\hat{c}_{0 G}^{\widetilde{B}} \cong \hat{\mathrm{c}}_{0}, \hat{c}_{G}^{\widetilde{B}} \cong \hat{\mathrm{c}}$ and $\hat{c} s_{G}^{\widetilde{B}} \cong \hat{\mathrm{c} s}$.

Proof. We prefer to prove just one because there are three similar results. The first thing to do in this proof is to show that a linear transformation exist between the spaces $\hat{c}_{G}^{\widetilde{B}}$ and $\hat{c}$. Namely, the required tool is to describe a transformation from $\hat{c}_{G}^{\widetilde{B}}$ to $\hat{c}$. Using the matrix $\widetilde{R}$ introduced in the second chapter, if the transformation $T$ is described as $T(x)=\widetilde{R} \cdot x$ for every $x \in \hat{c}_{G}^{\widetilde{B}}$, it is easy to see that $T$ is linear. If $T(x)=0$ then $x=0$, so $T$ is one-to-one. Finally, we need to show that $T$ is surjective. If $x=\left(x_{k}\right)$ is defined

$$
x_{k}=\sum_{j=0}^{k-1} \frac{1}{u_{j}} \Omega_{k}^{j} y_{j}+\frac{1}{u_{k} r_{k} v_{k}} y_{k} ;(k \in I N)
$$

all $y \in \hat{c}$. With this in mind, it is easy to see that the following equation is satisfied

$$
u_{k}\left[\sum_{j=0}^{k-1}\left(r_{j} v_{j}+s_{j} v_{j+1}\right) x_{j}+v_{k} x_{k} r_{k}\right]=y_{k}
$$

for all $k \in I N$, the following is true from here

$$
\begin{aligned}
\lim _{m \rightarrow \infty} \sum_{k=0}^{m} \frac{u_{n+k}}{m+1}\left[\sum_{i=0}^{n+k-1} k_{i} x_{i}+v_{k+n} r_{k+n} x_{k+n}\right] & =\lim _{m \rightarrow \infty} \sum_{k=0}^{m} y_{k+n} \\
& =\hat{c}-\lim y_{k} .
\end{aligned}
$$

This means that $x=\left(x_{k}\right) \in \hat{c}_{G}^{\widetilde{B}}$. In other words, $T$ is surjective. Therefore, $T$ is a linear bijection which means that the spaces $\hat{c}_{G}^{\widetilde{B}}$ and $\hat{c}$ are linear isomorphic.

As it is well-known that the matrix domain $\chi_{A}$ of the normed sequence space $\chi$ has a basis 
if and only if $\chi$ has a basis whenever $A=\left(a_{n k}\right)$ is a triangle and the almost convergent sequence space $\hat{c}$ has definitely not Schauder basis by (Remark 2.4) [14] , we have;

Corollary 2.4. The space $\hat{c}_{G}^{\widetilde{B}}$ has no Schauder basis.

\section{THE $\alpha-, \beta-$ and $\gamma$-DUALS of AFOREMENTIONED SPACES}

The intuition of multiplier space plays very crucial role in this section. In order to state $\alpha-$, $\beta$ - and $\gamma$-duals of the aforementioned sequence spaces, we are going to give the terminology a multiplier space.

The set $S(X, Y)$ is known as the multiplier space of a given sequence spaces $X$ and $Y$,

$$
S(X, Y)=\{z \in w: z x \in Y \text { for all } x \in Y\} .
$$

It can be note here that for any sequence space $\varphi$ satisfying both conditions with $X \subset \varphi$ and $\varphi \subset Y$ that is $\varphi$ including $X$ and included by $Y$, it is valid not only $S(X, Y) \subset S(X, \varphi)$ but also $S(X, Y) \subset S(\varphi, Y)$ holds, respectively.

When evaluating the multiplier space $S(X, Y)$, the $\alpha-, \beta-$ and $\gamma-$ duals of a sequence space $X$, which are respectively denoted by $X^{\alpha}$, $X^{\beta}$ and $X^{\gamma}$ are described by

$$
X^{\alpha}=S(X, Y), X^{\beta}=S(X, Y), X^{\gamma}=S(X, Y)
$$

By order, these duals are known by the names Köthe-Toeplitz dual, generalized Köthe-Toeplitz dual and Garling dual, respectively.
Let us now state the following lemmas. Because they will be necessary when we prove that we will.

Lemma 3.1. $A=\left(a_{n k}\right) \in\left(\hat{c}, l_{\infty}\right)$ if and only if $\sup _{n} \sum_{k}\left|a_{n k}\right|<\infty \quad[15]$.

Lemma 3.2. $A=\left(a_{n k}\right) \in(\hat{c}, c)$ if and only if $\sup _{n} \sum_{k}\left|a_{n k}\right|<\infty$, and there are $\alpha_{k}, \alpha \in C$ in such a way that $\lim _{n \rightarrow \infty} a_{n k}=\alpha_{k}$ for any $k \in I N \lim _{n \rightarrow \infty} \sum_{k} a_{n k}=\alpha[17]$.

Theorem 3.3. The $\gamma-$ dual of the space $\hat{c}_{G}^{\widetilde{B}}$ is the intersection of the following two sets.

$$
f_{1}=\left\{a \in w: \sup _{n} \sum_{k=1}^{n-1}\left|\frac{1}{u_{k}} \sum_{j=k+1}^{n} \Omega_{k}^{j} a_{j}+\frac{1}{u_{k} r_{k} v_{k}}\right|<\infty\right\},
$$

$$
f_{2}=\left\{a \in w: \sup _{n}\left|\frac{a_{n}}{u_{n} r_{n} v_{n}}\right|<\infty\right\} .
$$

Proof. The required idea in this proof is to use the definition of the Köthe-Toeplitz dual. Suppose that $a=\left(a_{k}\right) \in w$. Under these assumptions, we are obviously able to get the following equalities;

$$
\begin{aligned}
& \begin{aligned}
\sum_{k=0}^{n} a_{k} x_{k} & =\sum_{k=0}^{n} a_{k}\left[A_{j}^{n}(\Omega)+\frac{1}{u_{k} r_{k} v_{k}} y_{k}\right] \\
& =\sum_{k=0}^{n-1}\left[A_{n}^{j}(\Omega)+\frac{a_{k}}{u_{k} r_{k} v_{k}}\right] y_{k}+\frac{a_{n}}{u_{n} r_{n} v_{n}} y_{n} \\
& =(F y)_{n}
\end{aligned} \\
& \text { where } A_{j}^{n}(\Omega)=\sum_{j=0}^{k-1} \frac{1}{u_{j}} \Omega_{k}^{j} y_{j} \text { and }
\end{aligned}
$$


$A_{n}^{j}(\Omega)=\frac{1}{u_{k}} \sum_{j=k+1}^{n} \Omega_{j}^{k} a_{j}$. Where $F=\left\{f_{n k}\right\}$ is

defined by

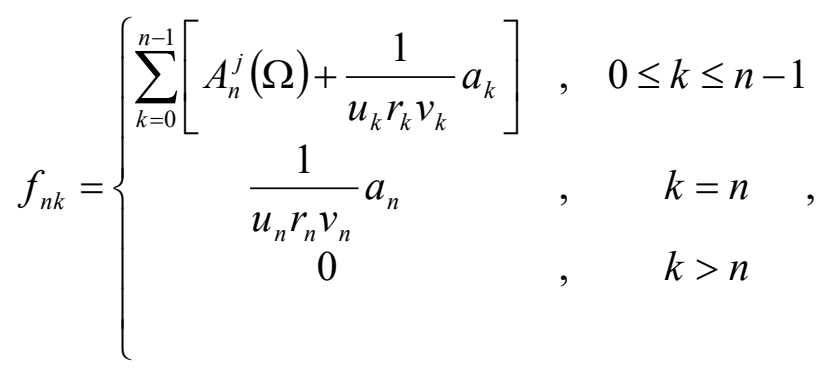

for a given $k, n \in I N$. Therefore, we observe from $\sum_{k=0}^{n} a_{k} x_{k}=(F y)_{n}$ that $a . x \in b s$ whenever $x \in \hat{c}_{G}^{\widetilde{B}}$ if and only if $F . y \in l_{\infty}$ whenever $y \in \hat{c}$. Thus, we derive with the aid of Lemma 3.1 $\left(\hat{c}_{G}^{\widetilde{B}}\right)^{\gamma}=f_{1} \cap f_{2}$, which gives the desired result.

Theorem 3.4. The generalized Köthe-Toeplitz dual of the space $\hat{c}_{G}^{\widetilde{B}}$ is the intersection of the following there sets

$$
\begin{gathered}
f_{3}=\left\{a \in w: \lim _{n \rightarrow \infty} f_{n k} \text { exists }\right\} \\
f_{4}=\left\{a \in w: \lim _{n \rightarrow \infty} \sum_{k} f_{n k} \text { exists }\right\}, \\
f_{5}=\left\{a \in w: \lim _{n \rightarrow \infty} \sum_{k} \Delta\left(f_{n k}-\alpha_{k}\right)<\infty\right\},
\end{gathered}
$$

where $\alpha_{k}=\lim _{n \rightarrow \infty} f_{n k}$.

Proof. The method for determining the generalized Köthe-Toeplitz dual of the space $\hat{c}_{G}^{\widetilde{B}}$ is based on the definition of $\beta$-dual introduced above. So, we will take the steps required by the relevant definition. Suppose that $a \in w$. In that case, we can easily deduce, form $\sum_{k=0}^{n} a_{k} x_{k}=(F y)_{n} \quad$ that $\quad a . x \in C S \quad$ whenever $x \in \hat{c}_{G}^{\widetilde{B}}$ if and only if $F . y \in c$ whenever $y \in \hat{c}$. Therefore, we obtain from Lemma 3.2. that $\left(\hat{c}_{G}^{\widetilde{B}}\right)^{\beta}=f_{3} \cap f_{4} \cap f_{5}$, which proves our assertion.

Theorem 3.5. The $\gamma$-dual of the space $\hat{c} s_{G}^{\widetilde{B}}$ is the intersection of the following sets;

$$
\begin{aligned}
& f_{6}=\left\{a \in w: \sup _{n} \sum_{k}\left|\Delta f_{n k}\right|<\infty\right\}, \\
& f_{7}=\left\{a \in w: \lim _{k \rightarrow \infty} f_{n k}=0\right\} .
\end{aligned}
$$

Namely $\left\{\hat{c} s_{G}^{\widetilde{B}}\right\}^{\beta}=f_{6} \cap f_{7}$.

Proof. The proof can be done a much similar way to proof of the Theorem 3.3, for this reason, it will not be repeated here.

Theorem 3.6. Define the set

$$
f_{8}=\left\{a \in w: \lim _{n \rightarrow \infty} \sum_{k}\left|\Delta^{2} f_{n k}\right|<\infty\right\} .
$$

Then $\left\{\hat{c} s_{G}^{\widetilde{B}}\right\}^{\beta}=f_{3} \cap f_{6} \cap f_{7} \cap f_{8}$.

Proof. Since the proof does not have any difficulty and even it is very similar to proof of Theorem 3.4, it can be proven.

\section{SOME MATRIX TRANSFORMATIONS}

In this final section, we characterize some matrix transformations.

We shall use representations following throughout all over the section for brevity that 
$a_{n k}=\sum_{j=0}^{n} a_{j k}, a(n, k, m)=\frac{1}{m+1} \sum_{j=0}^{m} a_{n+j, k} \quad$ and $\Delta a_{n k}=a_{n k}-a_{n, k+1}$.

We will be able to prove our main theorem after we have one more lemma with which to work.

Lemma 4.1. Let $\lambda$ be an $F K$ - space, $U$ be any triangle matrix, $V$ be its inverse matrix and $\mu$ be any subset of $w$. Then, we obtain $A=\left(a_{n k}\right) \in\left(\lambda_{U}: \mu\right)$ if and only if $C^{(n)}=\left(C_{m k}^{(n)}\right) \in(\lambda, c)$ for all $n \in I N$ $C=\left(c_{n k}\right) \in(\lambda: \mu)$,

where

$$
C_{m k}^{n}=\left\{\begin{array}{ccc}
\sum_{j=k}^{m} a_{n j} v_{j k} & , & 0 \leq k \leq m, \\
0, & k>m,
\end{array}\right.
$$

and $c_{n k}=\sum_{j=k}^{\infty} a_{n j} v_{j k}$ for all $k, m, n \in I N$ [16].

Lemma 4.2. An infinite matrix $A=\left(a_{n k}\right)$ transforms all almost convergent sequences into almost convergent sequences if and only if $\|A\|=\sup _{n} \sum_{k}\left|a_{n k}\right|<\infty, \quad \hat{c}-\lim _{n \rightarrow \infty} \sum_{k} a_{n k}=\alpha$ ， $\hat{c}-\lim _{n \rightarrow \infty} a_{n k}=\alpha_{k}$ for $\quad$ each $\quad k \in I N$, $\lim _{q \rightarrow \infty} \sum_{k} \frac{1}{q+1}\left|\sum_{i=0}^{q}\left(a_{n+i, k-1}-\alpha_{k-1}+\alpha_{k}-a_{n+i, k}\right)\right|=0$ uniformly in $n$ [17].

Let $A=\left(a_{n k}\right)$ be an infinite matrix. From now on, we will use the following notions;

$d_{m k}^{n}=\widetilde{a}_{n k}(m)=\frac{1}{u_{k} r_{k} v_{k}} a_{n k}+\frac{1}{u_{k}} \sum_{j=k+1}^{m} \Omega_{k}^{j} a_{n j}$ and $d_{n k}=\widetilde{a}_{n k}=\frac{1}{u_{k} r_{k} v_{k}} a_{n k}+\frac{1}{u_{k}} \sum_{j=k+1}^{\infty} \Omega_{k}^{j} a_{n j}$ for all $n, k, m \in I N$.

Theorem 4.3. Suppose that the entries of the infinite matrices given by $A=\left(a_{n k}\right)$ and $Z=\left(z_{n k}\right)$ are linked to the following connection

$$
z_{n k}=\widetilde{a}_{n k}
$$

for all $k, n \in I N$ and $\mu$ any given sequence space. Then, $A \in\left(\hat{c}_{G}^{\widetilde{B}}, \mu\right)$ if and only if $\left\{a_{n k}\right\}_{k \in I N} \in\left\{\hat{c}_{G}^{\widetilde{B}}\right\}^{\beta} \quad$ for $\quad$ all $\quad n \in I N \quad$ and $Z \in(\hat{c}: \mu)$.

Proof. Firts of all, assume that $\mu$ be a sequence space and take into account the condition $z_{n k}=\widetilde{a}_{n k}$ given above between the entries of the matrices $A$ and $Z$ and let us remember the fact that the newly defined sequence space $\hat{c}_{G}^{\widetilde{B}}$ and almost convergent sequence space $\hat{c}$ are linearly isomorphic from the Theorem 2.3.

In this part of the proof, our first job is to take both $A \in\left(\hat{c}_{G}^{\widetilde{B}}, \mu\right)$ and a given sequence $y \in \hat{c}$. Because of these assumptions, it is fairly easy to say that $Z . \widetilde{R}$ exists and $\left\{a_{n k}\right\}_{k \in I N} \in \bigcap_{n=1}^{5} f_{i}$. The newly obtained results requires that $\left\{z_{n k}\right\}_{k \in I N} \in l_{1}$ for each $n \in I N$. Thus, Z.y exists and therefore, $\quad \sum_{k} z_{n k} y_{k}=\sum_{k} a_{n k} x_{k}$ for all $n \in I N$. We obtain, with the aid of $z_{n k}=\widetilde{a}_{n k}$, which results in the fact that $Z \in(\hat{c}, \mu)$.

For proving the sufficiency of the claim, let $\left\{a_{n k}\right\}_{k \in I N} \in\left\{\hat{c}_{G}^{\widetilde{B}}\right\}^{\beta}$ for $\quad$ each $n \in I N \quad$ and 
$Z \in(\hat{c}, \mu)$ hold, and take a given sequence $x \in \hat{c}_{G}^{\widetilde{B}}$. By our assumption, it is not difficult to see that $A . x$ exists. Furthermore, we can easily see that $A . x=Z . y$ from the following rudimentary calculations

$$
\sum_{k=0}^{m} a_{n k} x_{k}=\sum_{k=0}^{m}\left[\sum_{j=0}^{k-1} \frac{1}{u_{j}} \Omega_{k}^{j} a_{n j} y_{j}+\frac{a_{n k} y_{k}}{u_{k} r_{k} v_{k}}\right]
$$

making $m \rightarrow \infty$ in this newly obtained formula. This result indicates that $A \in\left(\hat{c}_{G}^{\widetilde{B}}, \mu\right)$.

We obtain following theorem, by changing the roles of spaces $\hat{c}_{G}^{\widetilde{B}}$ and $\hat{c}$ with $\mu$.

Theorem 4.4. Suppose that the entries of the infinite matrices $A=\left(a_{n k}\right)$ and $B=\left(b_{n k}\right)$ are linked to the connection $b_{n k}=\hat{a}_{n k}$ for all $k, n \in I N$ and $\mu$ is a given sequence space. Then, $A \in\left(\mu, \hat{c}_{G}^{\widetilde{B}}\right)$ if and only if $B \in(\mu, \hat{c})$.

Proof. For this, assume that $x=\left(x_{k}\right) \in \mu$ and after then let us make the following fairly simple computation. Actually, the process of computation that $\{\widetilde{R} .(A \cdot x)\}_{n}=\{B . x\}_{n}$ is a little long but not difficult, so we omit the details. It is clearly seen that $A \cdot x \in \hat{c}_{G}^{\widetilde{B}}$ if and only if B. $x \in \hat{c}$. In fact, this is exactly what we want to prove.

It is time to present the conditions which are going to be used in the proofs:

$$
\sup _{n} \sum_{k}\left|a_{n k}\right|<\infty
$$

$\lim _{n \rightarrow \infty} a_{n k}=\alpha_{k}$, for each fixed $k \in I N$

$$
\begin{aligned}
& \lim _{n \rightarrow \infty} \sum_{k} a_{n k}=\alpha \\
& \lim _{n \rightarrow \infty} \sum_{k}\left|\Delta\left(a_{n k}-\alpha_{k}\right)\right|=0 \\
& \sup _{n} \sum_{k}\left(\Delta\left|a_{n k}\right|\right)<\infty \\
& \lim _{n \rightarrow \infty} a_{n k}=0, \text { for each fixed } n \in I N
\end{aligned}
$$

$\lim _{n \rightarrow \infty} \sum_{k}\left|\Delta^{2} a_{n k}\right|=\alpha$

$\hat{c}-\lim a_{n k}=\alpha_{k}$ exists, for each fixed $k \in I N$ [4.8]

$\lim _{m \rightarrow \infty} \sum_{k}\left|a(n, k, m)-\alpha_{k}\right|=0$, uniformly in $n$ [4.9]

$\hat{c}-\lim \sum_{k} a_{n k}=\alpha$

$\lim _{m \rightarrow \infty} \sum_{k}\left|\Delta a(n, k, m)-\alpha_{k}\right|=0$, unif. in $n$

$\lim _{q \rightarrow \infty} \sum_{k} \frac{1}{q+1}\left|\sum_{i=0}^{q}\left[\Delta a(n+i, k)-\alpha_{k}\right]\right|=0$

uniformly in $n$

$\sup _{n} \sum_{k}|\Delta a(n, k)|<\infty$

$\hat{c}-\lim a(n, k)=\alpha_{k}$ exists, for each fixed $k \in I N$

$\lim _{q \rightarrow \infty} \sum_{k} \frac{1}{q+1} \mid \sum_{i=0}^{q}\left[\Delta^{2} a(n+i, k)-\alpha_{k}\right]=0$

uniformly in $n$

$\sup _{n} \sum_{k}|a(n, k)|<\infty$ 
$\sum_{n} a_{n k}=\alpha_{k}$, for every fixed $k \in I N$

$\sum_{n} \sum_{k} a_{n k}=\alpha$

$\lim _{n \rightarrow \infty} \sum_{k}\left|\Delta a(n, k)-\alpha_{k}\right|=0$

Let $A=\left(a_{n k}\right)$ is an infinite matrix. Thus, the subsequent expressions retain.

Lemma 4.5. i) $A=\left(a_{n k}\right) \in\left(l_{\infty}: \hat{c}\right)$ iff [4.1], [4.8] and [4.9] hold [17].

ii) $A=\left(a_{n k}\right) \in(\hat{c}: \hat{c})$ iff [4.1], [4.8],[4.9] and [4.10] hold [17].

iii) $A=\left(a_{n k}\right) \in\left(\hat{c} s: l_{\infty}\right)$ iff [4.5], [4.6] hold [16].

iv) $A=\left(a_{n k}\right) \in(\hat{c} s: c)$ iff [4.2], [4.5] and [4.7] hold [18].

v) $A=\left(a_{n k}\right) \in(c: \hat{c})$ iff [4.1], [4.8] and [4.10] hold [19].

vi) $A=\left(a_{n k}\right) \in(b s: \hat{c})$ iff [4.5], [4.6], [4.8] and [4.12] hold [20].

vii) $A=\left(a_{n k}\right) \in(\hat{c} s: \hat{c})$ iff [4.6], [4.8], [4.11] and [4.12] hold [21].

viii) $A=\left(a_{n k}\right) \in(c s: \hat{c})$ iff [4.5] and [4.8] hold [22].

ix) $A=\left(a_{n k}\right) \in(b s: \hat{c} s)$ iff [4.6], [4.12], and [4.14] hold [20].

х) $A=\left(a_{n k}\right) \in(\hat{c} s: \hat{c} s)$ iff [4.12] and [4.15] hold [21]. xi) $A=\left(a_{n k}\right) \in(c s: \hat{c} s)$ iff [4.13] and [4.14] hold [22].

xii) $A=\left(a_{n k}\right) \in(\hat{c}: c s)$ iff [4.16] and [4.19] hold [23].

Corollary 4.6. The following statements hold:

$A=\left(a_{n k}\right) \in\left(\hat{c}_{G}^{\widetilde{B}}: l_{\infty}\right)$ iff $\left\{a_{n k}\right\}_{k \in I N} \in\left(\hat{c}_{G}^{\widetilde{B}}\right)^{\beta}$ for all $n \in I N$ and [4.1] holds with $\widetilde{a}_{n k}$ in place of $a_{n k}$.

$A=\left(a_{n k}\right) \in\left(\hat{c}_{G}^{\widetilde{B}}: c\right)$ iff $\left\{a_{n k}\right\}_{k \in I N} \in\left(\hat{c}_{G}^{\widetilde{B}}\right)^{\beta}$ for all $n \in I N$ and [4.1], [4.2] and [4.4] hold with $\widetilde{a}_{n k}$ in place of $a_{n k}$.

$A=\left(a_{n k}\right) \in\left(\hat{c}_{G}^{\widetilde{B}}: b s\right)$ iff $\left\{a_{n k}\right\}_{k \in I N} \in\left(\hat{c}_{G}^{\widetilde{B}}\right)^{\beta}$ for all $n \in I N$ and [4.16] holds with $\widetilde{a}_{n k}$ in place of $a_{n k}$.

$A=\left(a_{n k}\right) \in\left(\hat{c}_{G}^{\widetilde{B}}: c s\right)$ iff $\left\{a_{n k}\right\}_{k \in I N} \in\left(\hat{c}_{G}^{\widetilde{B}}\right)^{\beta}$ for all $n \in I N$ and [4.16] and [4.19] hold with $\widetilde{a}_{n k}$ in place of $a_{n k}$.

Corollary 4.7. The following statements are valid.

$A=\left(a_{n k}\right) \in\left(l_{\infty}, \hat{c}_{G}^{\widetilde{B}}\right)$ iff [4.1], [4.8] and [4.9] hold with $\hat{a}_{n k}$ in place of $a_{n k}$.

$A=\left(a_{n k}\right) \in\left(\hat{c}, \hat{c}_{G}^{\widetilde{B}}\right)$ iff [4.1], [4.8], [4.10] and [4.11] hold with $\hat{a}_{n k}$ in place of $a_{n k}$.

$A=\left(a_{n k}\right) \in\left(c, \hat{c}_{G}^{\widetilde{B}}\right)$ iff [4.1], [4.8] and [4.10] hold with $\hat{a}_{n k}$ in place of $a_{n k}$.

Corollary 4.8. The following expression retain: 
$A=\left(a_{n k}\right) \in\left(b s, \hat{c}_{G}^{\widetilde{B}}\right)$ iff [4.5], [4.6], [4.8] and [4.9] hold with $\hat{a}_{n k}$ in place of $a_{n k}$.

$A=\left(a_{n k}\right) \in\left(\hat{c} s, \hat{c}_{G}^{\widetilde{B}}\right)$ iff [4.6], [4.8] and [4.13] hold with $\hat{a}_{n k}$ in place of $a_{n k}$.

$A=\left(a_{n k}\right) \in\left(c s, \hat{c}_{G}^{\widetilde{B}}\right)$ iff [4.5] and [4.8] hold with $\hat{a}_{n k}$ in place of $a_{n k}$.

Corollary 4.9. The following statements hold:

\section{REFERENCES}

[1] Choudhary B., Nanda S. Functional Analysis with applications, John Wiley and Sons, (1989), New Delhi, İndia.

[2] Candan M., A new sequence space isomorphic to the space $\ell(p)$ and compact operators, J. Math. Comput. Sci.,4-2 (2014) 306-334.

[3] Lorentz G. G. A contribution to the theory of divergent sequences, Acta Mathematica, 80 (1948) 167-190.

[4] Kizmaz H. On certain sequence spaces, Canad. Math. Bull. 24-2 (1981) 169-176.

[5] Kirişçi M., Almost convergence and generalized weighted mean. AIP Conf. Proc. 1470 (2012) 191-194.

[6] Başar F., Kirişçi M. Almost convergence and generalized difference matrix, Comput. Math. Appl.,61 (2011) 602-611.

[7] Kayaduman K., Şengönül M. The space of Cesaro almost convergent sequence and core theorems, Acta Math. Scientia,6 (2012) 2265-2278 .

[8] Candan M. Almost convergence and double sequential band matrix, Acta Math. Scientia, 34-2 (2014): 354-366.

[9] Candan M., Kılınç G. A different look for paranormed Riesz sequence space derived by Fibonacci Matrix. Konuralp Journal of Mathematics 3-2 (2015) 62-76.
$A=\left(a_{n k}\right) \in\left(b s, \hat{c} s_{G}^{\widetilde{B}}\right)$ iff [4.6], [4.12] and [4.14] retain with $\hat{a}_{n k}$ in place of $a_{n k}$.

$A=\left(a_{n k}\right) \in\left(\hat{c} s, \hat{c} s_{G}^{\widetilde{B}}\right)$ iff [4.12] and [4.15] retain with $\hat{a}_{n k}$ in place of $a_{n k}$.

$A=\left(a_{n k}\right) \in\left(c s, \hat{c} s_{G}^{\widetilde{B}}\right)$ iff [4.13] and [4.14] retain with $\hat{a}_{n k}$ in place of $a_{n k}$.

[10] Kirişçi M. Almost convergence and generalized weighted mean II. J. Ineq. and Appl., 1-93 (2014) 13 pages.

[11] Polat H., Karakaya V., Şimşek N. Difference sequence space derived by using a generalized weighted mean. Applied Mathematics Letters, 24 (2011) 608-614.

[12] Karaisa A., Başar F. Some new paranormed sequence spaces and core theorems. AIP Conf. Proc., 1611 (2014) 380-391.

[13] Karaisa A., Özger F. Almost difference sequence spaces derived by using a generalized weighted mean, J. Comput. Anal. and Appl., 19-1 (2015) 27-38.

[14] Jarrah A. M., Malkowsky E. BK- spaces, bases and linear operators, Ren. Circ. Mat. Palermo, 52-2 (1990) 177-191.

[15] Siddıqi J. A. Infinite matrices summing every almost periodic sequences, Pacific J. Math., 39-1 (1971) 235-251.

[16] Başar F. Summability Theory and Its Applications, Bentham Science Publishers. e-books, Monographs, xi +405 pp., (2012) İstanbul, ISB:978-1-60805252-3.

[17] Duran J. P. Infinite matrices and almost convergence. Math. Z., 128 (1972) 75-83.

[18] Öztürk E. On strongly regular dual summability methods, Commun. Fac. Sci. 
Univ. Ank. Ser. Aâ, Math. Stat.,32 (1983) $1-5$.

[19] King J. P. Almost summable sequences. Proc. Amer. Math. Soc., 17 (1966) 12191225.

[20] Başar F., Solak İ. Almost-coercive matrix transformations, Rend. Mat. Appl., 11-2 (1991) 249-256.

[21] Başar F. f-conservative matrix sequences, Tamkang J. Math., 22-1 (1991) 205-212.

[22] Başar F., Çolak R. Almost conservative matrix transformations, Turkish J. Math., 13-3 (1989) 91-100.

[23] Başar F. Strongly-conservative sequence to series matrix transformations, Erc.Üni. Fen Bil. Derg., 5-12 (1989) 888-893.

[24] Candan M., Kayaduman K. Almost Convergent Sequence Space Derived By Generalized Fibonacci Matrix and Fibonacci Core,British J. Math. Comput. Sci, 7-2 (2015) 150-167.

[25] Başarır M., Başar F., Kara E. E. On The Spaces Of Fibonacci Difference Null And Convergent Sequences, arXiv:1309.0150v1 (2013) [math.FA].

[26] Kara E. E., Some topological and geometrical properties of new Banach sequences, J. Inequal. Appl., 38 (2013) 15 pp.

[27] Candan M. A new aproach on the spaces of generalized Fibonacci difference null and convergent sequences. Math. Aeterna, 1-5 (2015) 191-210.

[28] Candan M and Kara E. E., A study on topolojical and geometrical characteristics of new Banach sequence spaces, Gulf J. of Math., 3-4 ( 2015) 67-84.

[29] Kara E. E., Başarır M. On compact operators and some Euler$B^{(m)}$-difference sequence spaces, J. Math. Anal. Appl., 379-2 (2011) 499-511.

[30] Başarır M., Kara E. E. On some difference sequence spaces of weighted means and compact operators, Ann. Funct. Anal., 2-2 (2011) 114-129.

[31] Başarır M., Kara E. E. On the B-difference sequence space derived by generalized weighted mean and compact operators, J. Math. Anal. Appl., 391-1 (2012) 67-81.

[32] Kara E. E., İlkhan M. Some properties of generalized Fibonacci sequence spaces, Linear Multilinear Algebra, 64-11 (2016) 2208-2223.

[33] Kara E. E., Ilkhan M. On Some Banach Sequence Spaces Derived by a New Band Matrix, Br. J. Math. Comput. Sci., 9-2 (2015) 141-159 .

[34] Demiriz S., Kara E. E., Başarır M. On the Fibonacci Almost Convergent Sequence Space and Fibonacci Core, Kyungpook Math. J., 55-2 (2015) 355-372.

[35] Kara E. E., Demiriz S. Some New Paranormed Difference Sequence Spaces Derived by Fibonacci Numbers, Miskolc Math. Notes, 16-2 (2015) 907-92.

[36] Kara E. E., Başarır M., Mursaleen M. Compactness of matrix operators on some sequence spaces derived by Fibonacci numbers”, Kragujevac J. Math., 39-2 (2015) 217-230.

[37] Kirişçi M. Reisz type integrated and differentiated sequence spaces, Bulletin of Mathematical Analysis and Applications, 7 (2015) 14-27.

[38] Kirişçi M. A note on the some geometric properties of the sequence spaces defined by Taylor method, Far East Journal of Mathematical Sciences (FJMS), 102-7 (2017) 1533-1543.

[39] Kirişçi M. Integrated and differentiated spaces of triangular fuzzy numbers", Fasciculi Mathematici, 59 (2017), 75-89.

[40] Kirişçi M. Integrated and Differentiated Sequence Spaces, Journal Nonlinear Analysis and Application, (2015 2-16.

[41] Kirişçi M. On the Taylor sequence spaces of nonabsolute type which include the 
spaces $c_{0}$ and $c$, J. of Math. Analy., 6 (2015) 22-35.

[42] Kirişçi M. P-Hahn sequence space", Far East Journal of Mathematical Sciences, 90 (2014) 45-63.

[43] Kirişçi M. The Sequence Space bv and Some Applications, Mathematica Aeterna, 4 (2014) 207-223.

[44] Kirişçi M. On the spaces of Euler almost bull and Euler almost convergen sequences", Communications, Series A1:Mathematics and Statistics, 62 (2013) 85-100.

[45] Ercan S., Bektaş Ç. A. On new $\lambda^{2}$ - Convergent Difference BK-spaces, J. Comput. Anal. Appl., 23-5 (2017) 793801.

[46] Ercan S., Bektaş Ç. A. Some topological and geometric properties of a new BKspace derived by using regular matrix of Fibonacci numbers. Linear Multi linear Algebra, 65-5 (2017) 909-921.

[47] Ercan S. and Bektaş Ç. A., The dual spaces of new $\lambda^{m}$-sequence spaces and their matrix maps. AIP Conference Proceedings, 1798-1 (2017). AIP. Publishing.

[48] Ercan S.,Bektaş Ç. A. New properties of BK-spaces defined by using regular matrix of Fibonacci numbers. AIP Conference Proceedings, 1738-1 (2016), AIP. Publishing.

[49] Ercan S.,Bektaş Ç. A. On the spaces of $\lambda^{m}$-bounded and $\lambda^{m}$-absolutely $p$-summable sequences. Facta Univ. Ser. Math. Inform., 32- 3 (2017) 303-318.

[50] Et M., Karakaş M., Çınar M. Some Geometric Properties of a New Modular Space, Defined by Zweier Operator, Fixed Point Theory and Applications, 165 (2013) $10 \mathrm{pp}$.

[51] Karakaş M., Karabudak H. Lucas Sayıları ve Sonsuz Toeplitz Matrisleri Üzerine Bir Uygulama, Cumhuriyet Sci. J.,38-3 (2017) 557-562.

[52] Karakaş M., Karakaş A. M. New Banach Sequence Spaces That Is Defined By the Aid of Lucas Numbers, Iğdır Univ. J. Inst. Sci. \&amp; Tech., 7-4 (2017) 103-111.

[53] Kilınç G., Candan M. A different approach for almost sequence spaces defined by a generalized weighted mean. SAU, 21-6 (2017) 1529-1536. 